\title{
Análise Comprobatória teórico-prática da semelhança entre dimensões para detecção de Estilos de Aprendizagem impactando na ausência de resultados significativos
}

Andreia Rosangela Kessler Mühlbeier - UNICRUZ - andreiamuhlbeier@yahoo.com.br Leander Cordeiro de Oliveira - UNICRUZ - leander_dewon@yahoo.com.br. Patrícia Mariotto Mozzaquatro - UNICRUZ - patriciamozzaquatro@gmail.com Rosecleia Duarte Medina - UFSM - roseclea.medina@gmail.com

\section{Resumo}

Este artigo apresenta uma Análise Comprobatória teórico-prática apresentando a semelhança entre as diferentes dimensões dos estilos de aprendizagem, mostrando que a utilização de todos os métodos não gerou impactos significativos nos resultados encontrados. A pesquisa embasou-se nos resultados de uma dissertação de mestrado e um trabalho de conclusão de curso que trabalharam com os modelos de diferentes autores e que foram validadas por docentes e discentes de cursos de Graduação e Pós- Graduação. Após a análise pode-se comprovar a semelhança entre várias dimensões e uma tendência de mesclar estilos o que confirma as conclusões de diferentes autores em relação a alguns destes estilos estarem imbricados.

Palavras-chave: Estilos de aprendizagem, AVAs adaptados, Análise de estilos.

\section{Analysis proving theoretical and practical of similarity between dimensions for detection of Learning Styles impacting in the abs ence of significant results}

\begin{abstract}
This paper presents a theoretical and pratical evidentiary analysis showing the similarity between the different dimensions of learning styles, showing that the use of all methods did not generate significant impacts on results. The underlying research on the results of a dissertation and a final paper of course worked with models of different authors and were validated by teachers and students of undergraduate and graduate. After analysis we can prove the similarity between various dimensions and a tendency to merge styles which confirms the conclusions of different authors for some of these styles are interwoven.
\end{abstract}

Keywords: Learning styles, Adapted AVAs, Styles analysis.

\section{Introdução}

No momento educacional vivido atualmente, pretende-se a centralização da aprendizagem pelo próprio aluno. Suas experiências e interesses são peças fundamentais neste contexto e a identificação, assim como saber tirar proveito do conhecimento dos estilos de aprendizagem de cada um pode constituir um ótimo preceito para garantir o sucesso acadêmico destes alunos (Witkin e Goodenough 1981).

Segundo Pennings e Span (1991), os estilos de aprendizagem "se relacionam às preferências dos sujeitos em relação às estratégias de aprendizagem na construção do conhecimento". Mas a quantidade de estilos existentes identificados por teóricos é elevada. Segundo alguns autores não é preciso se trabahhar com todas as dimensões, mas as pesquisas 
não definem uma diferenciação muito exata entre estes estilos de aprendizagem e nem sua quantidade definitiva.

Através destes estudos, surgem alguns questionamentos relevantes: a utilização de um grande número de estilos na detecção do perfil do aluno pode ser um empecilho na definição da sua maneira de aprender? Quais estilos seriam adotados na adaptação de um ambiente virtual de aprendizagem?

A pesquisa desenvolvida justifica-se devido a questionamentos elencados nos trabalhos de (MOZZAQUATRO, 2010) e (MÜHLBEIER, 2010), objetivando detectar quais dimensões são mais adequadas para serem implementadas em ambientes virtuais de aprendizagem.

$\mathrm{O}$ artigo aborda a semelhança entre as diferentes dimensões de estilos de aprendizagem como também os estilos imbricados, comprovando de maneira prática as teorias através de um estudo integrando a dissertação de mestrado intitulada "Adaptação do Mobile Learning Engine Moodle (MLE Moodle) aos Diferentes Estilos Cognitivos Utilizando Hipermídia Adaptativa" (MOZZAQUATRO, 2010) e o trabalho de conclusão de curso "Estilos e Estratégias de Aprendizagem Personalizadas a Alunos das Modalidades Presenciais e a Distância" (MÜHLBEIER, 2010).

\section{Estilos de Aprendizagem}

Aprendizagem pode ser conceituada como um processo natural na vida de todo ser humano e concluído na idade adulta. Pode-se perceber que pessoas da mesma classe, idade, nacionalidade, raça e/ou religião preferem aprender de maneiras diferentes, ou seja tem a sua própria preferência no momento de aprender.

Preferência ou estilo de aprendizagem é o modo como cada aprendiz se concentra, processa e retém informação. As características pessoais fazem com que o mesmo método de ensino seja efetivo para alguns e ineficaz para outros. (DUNN, 1989).

Sabe-se que identificar os estilos de aprendizagem dos alunos é importante, uma vez que pode explicar o porquê de certas estratégias de aprendizagem funcionarem bem e outras não.

De acordo com Cavellucci (2006, p. 10-11):

Os estilos de aprendizagem são a maneira com que o aprendiz utiliza estratégias de aprendizagem na construção do conhecimento. Tais estratégias são ferramentas que o sujeito desenvolve para lidar com diferentes situações de aprendizagem incompatíveis com seu estilo.

Portanto, quanto mais estratégias o aluno tiver desenvolvido, maior será a chance de lidar com as diversas formas de apresentação das informações nas situações de aprendizagem por ele vivenciadas.

Deve ser ressaltar que em pesquisas realizadas como (Mozzaquatro, 2010), (Cavellucci, 2006) e (Lopes, 2002):

Possuem propriedades adaptativas, uma vez que na interação como ambiente são adquiridas estruturas de conhecimento utilizadas na regulação dos comportamentos; estão relacionados com essas funções: motricidade, atenção, percepção, aprendizagem, memória, pensamento e resolução de problemas; a individualidade poderá ser caracterizada por padrões de adaptação típicos (estilos); os diferentes estilos são produtos da utilização de estratégias diferentes em tarefas e condições adaptativas semelhantes; as pessoas são mais eficientes em tarefas diárias que evocam sua capacidade 
adaptativa e que evidenciam seu estilo dominante; uma adaptação a longo prazo é otimizada se for complementada por modificações do estilo nãodominante. (LOPES, 2002, p.49)

Partindo das investigações realizadas acredita-se que seja adequado utilizar o termo preferências de aprendizagem para denominar o conjunto de preferências que determina uma abordagem individual para aprender.

\subsection{Dimensões}

Quanto às dimensões dos estilos de aprendizagem, um grande número delas encontra-se disponível na literatura. Podem ser citadas as seguintes, de maior relevância.

\subsubsection{Modelo Butler}

O modelo de Butler apresenta cinco dimensões de estilos de aprendizagem: Realista, Analítico, Pragmático, Pessoal e Divergente.

De acordo com Butler (2003, p.7): Estilo de aprendizagem é a forma consistente e pessoal através da qual as pessoas usam suas qualidades e habilidades para definir a si mesmas, para encontrar, avaliar e processar informações.

\subsubsection{Modelo Honey - Mumford}

Honey e Mumford (2000) consideraram que as principais características das pessoas podem ser integradas em quatro estilos de aprendizagem: Ativo, Reflexivo, Teórico e Pragmático. Admitem, ainda, que em cada pessoa é possível identificar características dos vários estilos de aprendizagem, embora geralmente, cada pessoa possua um estilo dominante.

\subsubsection{Modelo Felder e Silvermann}

Richard Felder compreende os estilos de aprendizagem como preferências e características dominantes no modo como as pessoas recebem e processam as informações. Para ele, "os estilos de aprendizagem são habilidades que podem e devem ser desenvolvidas no sujeito". (FELDER, 1993, p. 288).

\subsubsection{Modelo Kolb}

Kolb (1984) observa que cada sujeito desenvolve um estilo de aprendizagem particular, dando prioridade a certa habilidade. A partir da combinação das quatro habilidades descritas, ele propõe uma categoria dos estilos de aprendizagem: divergente, assimilador, convergente, acomodados.

\subsubsection{Modelo Bariani}

Bariani (1998) observa que as formas relativamente estáveis referentes às características da estrutura cognitiva de uma pessoa são modificadas a partir da influência direta ou indiretamente de novos eventos, através da cultura e de relacionar os dados da realidade elaborando conclusões sobre eles.

A Tabela 1 apresenta as principais características dos integrantes associados a cada um dos estilos conforme o seu autor.

Tabela 1 - Dimensões dos Estilos de Aprendizagem

\begin{tabular}{l|l|ll}
\hline & Dimensão & Características \\
\hline
\end{tabular}




\begin{tabular}{|c|c|c|}
\hline 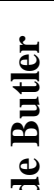 & Realista & $\begin{array}{l}\text { Os integrantes com estilo de aprendizagem realista vêem o mundo como um lugar } \\
\text { ordenado e previsível de fatos, ações e resultados. Eles são guiados pela experiência; } \\
\text { seguem orientações e certificam-se de que os outros estejam conscientes das ações que } \\
\text { praticaram; não gostam de mudar por mudar ou consertar o que não está com } \\
\text { problemas. }\end{array}$ \\
\hline$\frac{0}{\frac{0}{0}}$ & Analítico & $\begin{array}{l}\text { Os integrantes do estilo de aprendizagem analítico percebem o mundo como um } \\
\text { sistema lógico que pode ser compreendido através de análise e est udo constante; têm } \\
\text { uma teoria para quase tudo; gostam do método científico, informações técnicas e } \\
\text { provas. }\end{array}$ \\
\hline
\end{tabular}

\begin{tabular}{|c|c|c|}
\hline & Pragmático & $\begin{array}{l}\text { Os integrantes pragmáticos vêem o mundo do todo para as partes e das partes para o } \\
\text { todo, simultaneamente; têm habilidade de adaptar e adaptar-se; atribuem o mesmo } \\
\text { peso para fatos e valores; formulam estratégias e táticas para que as coisas aconteçam; } \\
\text { sofrem menos com as incompatibilidades de estilos. }\end{array}$ \\
\hline & Pessoal & $\begin{array}{l}\text { Os integrantes com estilo pessoal apresentam as seguintes características: entendem o } \\
\text { mundo como um lugar em que a harmonia é essencial e a prevalência cabe ao bem. } \\
\text { Estes sujeitos gostam de ser vistos como prestativos, apoiadores, abertos e dignos de } \\
\text { confiança; acolhem vários pontos de vista para depois assimilar; sentem-se } \\
\text { sobrecarregados e ignorados quando estão cercados de exigências normativas e lineares. }\end{array}$ \\
\hline & Divergente & $\begin{array}{l}\text { Os divergentes percebem o mundo como um lugar de infinitas possibilidades, onde } \\
\text { imaginação e experimentação se combinam para testar o que existe e descobrir o que } \\
\text { pode vir a ser; gostam de mudança; buscam discordância e novidade. }\end{array}$ \\
\hline \multirow{4}{*}{ 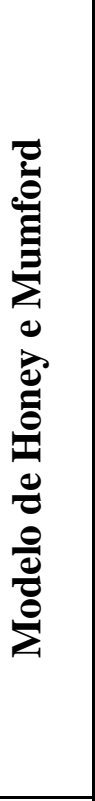 } & Ativo & $\begin{array}{l}\text { Preferem novas experiências, tem uma mente aberta, entusiasmam-se com qualquer } \\
\text { coisa nova, são sociáveis e envolvem-se constantemente com os outros, procuram ser } \\
\text { o centro de todas as atividades, interessam-se por desafios e situações problemáticas, } \\
\text { manifestam forte implicação na ação. }\end{array}$ \\
\hline & Reflexivo & $\begin{array}{l}\text { Dão prioridade a observação antes da ação, gostam de observar as experiências de } \\
\text { diversas perspectivas, centram-se na reflexão e na construção de significados, } \\
\text { recolhem informações tanto da sua própria experiência como da experiência dos } \\
\text { outros, preferem pensar antes de chegar a qualquer conclusão, gostam de observar os } \\
\text { outros em ação e de perceber o sentido geral da discussão antes de dizerem o que está } \\
\text { na sua própria mente. }\end{array}$ \\
\hline & Teórico & $\begin{array}{l}\text { Tendem a estabelecer relações, deduzir, integrar os fatos, em teorias coerentes, tendem } \\
\text { a ser perfeccionistas, gostam de analisar e de sintetizar. A sua abordagem aos } \\
\text { problemas é consistente e lógica. Procuram a racionalidade e a objetividade, sentem-se } \\
\text { desconfortáveis com conclusões subjetivas, pensamentos laterais ou qualquer aspecto } \\
\text { superficial. }\end{array}$ \\
\hline & Pragmático & $\begin{array}{l}\text { Gostam muito de experimentar idéias, teorias e técnicas para ver se funcionam na } \\
\text { prática. O seu ponto forte é a aplicação das idéias. Atuam de forma confiante e rápida } \\
\text { sobre as idéias e os projetos que os atraem, tendem a evitar a reflexão e ficam } \\
\text { impacientes com discussões sem fim. São pessoas práticas, gostam de chegar a } \\
\text { conclusões práticas e de resolver problemas. }\end{array}$ \\
\hline \multirow{4}{*}{ 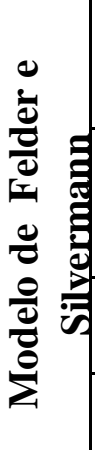 } & Sensorial & $\begin{array}{l}\text { Integrantes sensoriais gostam de aprender fatos; resolver problemas com métodos } \\
\text { estabelecidos, sem complicações e surpresas; são mais detalhistas e saem-se bem em } \\
\text { trabalhos práticos (em laboratório, por exemplo). }\end{array}$ \\
\hline & Intuitivo & $\begin{array}{l}\text { Integrantes intuitivos preferem descobrir possibilidades e relações; gostam de novidade } \\
\text { e se aborrecem com a repetição; sentem-se mais confortáveis para lidar com novos } \\
\text { conceitos, abstrações e fórmulas matemáticas; são mais rápidos no trabalho e mais } \\
\text { inovadores. }\end{array}$ \\
\hline & Visual & $\begin{array}{l}\text { Integrantes visuais lembram mais do que vêem - figuras, diagramas, fluxogramas, } \\
\text { filmes e demonstrações. }\end{array}$ \\
\hline & Verbal & $\begin{array}{l}\text { Integrantes verbais tiram maior proveito das palavras - explicações orais ou escritas e } \\
\text { fórmulas matemáticas. }\end{array}$ \\
\hline
\end{tabular}




\begin{tabular}{|c|c|c|}
\hline & Ativo & $\begin{array}{l}\text { Integrantes ativos tendem a compreender e reter informações mais eficientemente } \\
\text { discutindo, aplicando conceitos e/ou explicando para outras pessoas; gostam de } \\
\text { trabalhar em grupos. }\end{array}$ \\
\hline & Reflexivo & $\begin{array}{l}\text { Integrantes reflexivos precisam de um tempo para refletir sobre as informações } \\
\text { recebidas; preferem os trabalhos individuais. }\end{array}$ \\
\hline & Sequencial & $\begin{array}{l}\text { Integrantes sequenciais preferem aprender de forma linear, em etapas logicamente } \\
\text { sequenciadas; tendem a seguir caminhos lógicos para encontrar soluções. }\end{array}$ \\
\hline & Global & $\begin{array}{l}\text { Integrantes globais tendem a aprender de forma aleatória, formando uma visão do } \\
\text { todo; são hábeis para resolver problemas complexos com rapidez, mas têm dificuldade } \\
\text { para explicar como fizeram. }\end{array}$ \\
\hline \multirow{4}{*}{$\begin{array}{l}0 \\
0 \\
0 \\
\frac{1}{0} \\
\frac{0}{0} \\
\frac{0}{2}\end{array}$} & Divergente & $\begin{array}{l}\text { Integrantes divergentes preferem aprender pela experiência concreta e observação } \\
\text { reflexiva. Eles mostram-se habilidosos em situações que demandam idéias novas e } \\
\text { criativas; são capazes de analisar situações sob diferentes pontos de vistas e relacioná- } \\
\text { las em um todo organizado, compreendem as pessoas. Pergunta característica POR } \\
\text { QUÊ? }\end{array}$ \\
\hline & Assimilador & $\begin{array}{l}\text { Integrantes assimiladores aprendem por observação reflexiva e conceituação abstrata. } \\
\text { O sujeito assimilador utilizar raciocínio indutivo, responde à informação de forma } \\
\text { lógica e quando tem tempo pra refletir, tem facilidade para criar modelos abstratos e } \\
\text { teóricos e não se preocupa com o prático. Pergunta característica O QUÊ? }\end{array}$ \\
\hline & Convergente & $\begin{array}{l}\text { Integrantes convergentes usam raciocínio dedutivo, com aplicação prática das idéias, } \\
\text { aprendem por tentativa e erro, são hábeis para resolver problemas e tomar decisões. } \\
\text { Pergunta característica COMO? }\end{array}$ \\
\hline & Acomodador & $\begin{array}{l}\text { Integrantes desse estilo preferem a aprendizagem baseada na experimentação ativa e } \\
\text { na experiência concreta, adaptam-se a circunstâncias imediatas; gosta de desafios, atua } \\
\text { mais pelo que sente do que por uma análise lógica. Pergunta característica DE QUE } \\
\text { MANEIRA? }\end{array}$ \\
\hline \multirow{4}{*}{ 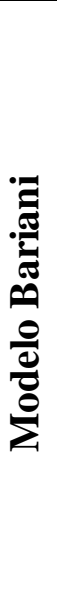 } & Reflexivo & $\begin{array}{l}\text { Refletem antes de empreender um determinado curso de ação, possuem os pensamentos } \\
\text { mais organizados. As pessoas cujos pensamentos são mais organizados, sequenciados e } \\
\text { que fazem ponderação préiva a uma resposta, são considerados reflexives. }\end{array}$ \\
\hline & Serealista & $\begin{array}{l}\text { Os sujeitos serialistas dão maior ênfase a tópicos separados e em sequencias lógicas, } \\
\text { buscando, posteriormente, padrões e relações no proceso, para confirmer ou não suas } \\
\text { hipóteses. Eles esclhem hipóteses mais simles e uma aboradagem lógico-linear (de uma } \\
\text { hipótese para a outra passo-a-passo). São muitas vezes bons analistas e hábeis para } \\
\text { resolver problemas. }\end{array}$ \\
\hline & Holista & $\begin{array}{l}\text { Os sujeitos holistas dão maior ênfase ao contexto global, desde o ínicio de uma tarefa, } \\
\text { preferem examiner uma grande quantidade de dados buscando padrões e relações entre } \\
\text { eles. Podem resolver rapidamente problemas complexos ou unir as coisas e são muitas } \\
\text { vezes bons sintetizadores. }\end{array}$ \\
\hline & Divergente & $\begin{array}{l}\text { Os divergentes percebem o mundo como um lugar de infinitas possibilidades, onde } \\
\text { imaginação e experimentação se combinam para testar o que existe e descobrir o que } \\
\text { pode vir a ser; gostam de mudança; buscam discordância e novidade. }\end{array}$ \\
\hline
\end{tabular}

Fonte: Mozzaquatro (2010).

$\mathrm{O}$ estilo de aprendizagem refere-se às preferências do sujeito no processo de aprendizagem. Partindo dos conceitos dos autores sobre estilos de aprendizagem, é possivel indenticar o seu próprio estilo.

\subsection{A Importância de diagnosticar o estilo de aprendizagem dos acadêmicos das modalidades presenciais e a distância}

No atual contexto pretende-se que o aluno seja o centro de sua própria aprendizagem, implementando-se um processo de aprendizagem significativa, em que a experiência e os interesses dos alunos são peças fundamentais, identificar e saber tirar 
proveito do conhecimento dos estilos de aprendizagem, pode constituir um ótimo princípio para garantir o sucesso acadêmico dos alunos.

Segundo (Geller, 2004), "o conhecimento do estilo de aprendizagem do aluno é importante para o professor que almeja a aprendizagem desse sujeito".

Uma combinação inadequada entre os estilos de aprendizagem dos alunos e as estratégias pedagógicas utilizadas pelo professor pode tornar os alunos entediados e desatentos, com resultados deficientes e, ademais, desanimados com o curso.

$\mathrm{O}$ autor (Hodgins, 2000) afirma que "a personalização de experiências de aprendizagem exige conhecer o aluno". O mesmo autor, acrescenta que quanto mais se conhecer o aluno para a construção do sistema de aprendizagem, maior é a oportunidade para the proporcionar informação adequada. A chave para o sucesso da aprendizagem é reconhecer que as diferenças de aprendizagem existem e que as mesmas devem ser reconhecidas, salientando que uma mesma abordagem não funciona do mesmo modo para todos os alunos (Palloff; Pratt, 2003).

\section{Descrição do experimento}

A pesquisa desenvolvida consistiu em uma análise comprobatória-teórico - prática da semelhança entre dimensões com o objetivo de detectar os estilos de aprendizagem. Conforme os autores (Riding; Cheema, 1991), (Geller, 2004), (Bariani, 1998) e (CAVELLUCCI, 2006, p.10) um aluno pode apresentar uma tendência a mesclar estilos de aprendizagem, sendo que a maioria das dimensões integrantes dos Modelos estudados: (WITKIN et al., 1981), (Riding; Rayner, 1998), (Bariani, 1998), (Felder - Silverman, 1993), (Honey; Munford, 2000), (Butler, 2003), (Kolb,1984) apresentam características semelhantes, confirmando o que os diferentes autores citados têm concluído em relação aos estilos de aprendizagem estarem imbricados.

O trabalho proposto buscou comprovar a semelhança entre as diferentes dimensões dos estilos de aprendizagem, comprovar que uma pessoa pode apresentar estilos imbricados, bem como detectar quais dimensões são mais adequadas para serem implementadas em um ambiente ambiente virtual de aprendizagem A pesquisa desenvolvida integrou uma dissertação de mestrado intitulada "Adaptação do Mobile Learning Engine Moodle (Mle Moodle) aos diferentes estilos cognitivos utilizando hipermídia adaptativa" (MOZZAQUATRO, 2010) e um trabalho de conclusão de curso intitulado "Estilos e Estratégias de Aprendizagem personalizadas a alunos das modalidades presenciais e a distância" (MÜHLBEIER, 2010).

$\mathrm{Na}$ pesquisa de (MOZZAQUATRO, 2010) foi adaptado o AVA móvel MLE Moodle aos diferentes estilos de aprendizagem utilizando técnicas de hipermídia adaptativa. Foi criado e aplicado um Sistema Especialista para diagnosticar os estilos, SEDECA. O estilo de aprendizagem foi identificado através do Instrumento "Questionário" embasado nos instrumentos de investigação propostos por (Felder, 1993), (Honey:Mumfor, 2000) e (Bariani, 1998). A autora trabalhou com quatro dimensões dos estilos de aprendizagem: Reflexivo, Serialista, Holista e Divergente a fim de subsidiar o processo de modelagem e implementação do AVA a ser adaptado. O questionário foi aplicado aos alunos dos cursos de Graduação e Pós- Graduação da modalidade EAD. 
A adaptação do conteúdo baseou-se em estereótipos, classificando os alunos de acordo com os estilos preferenciais. Para cada um destes estilos, foram definidas as melhores formas de apresentação de conteúdo, baseadas nos trabalhos de (Bariani, 1998) e (Geller, 2004). Através desse modelo, foram estabelecidas catorze técnicas de adaptação, sendo nove de conteúdo e cinco de avaliação, e definidas quais as mais adequadas para cada estilo cognitivo.

O AVA móvel adaptado foi validado por vinte e cinco alunos dos Cursos de Graduação e Pós Graduação a Distância do Sistema Universidade Aberta do Brasil (UAB). Foi ministrado um Curso sobre Softwares Educativos integrando uma população de cinquenta alunos.

O material integrante do Curso foi apresentado de acordo com os quatro estilos cognitivos: Holista, Serialista, Divergente e Reflexivo. As atividades existentes foram diferenciadas de acordo com os estilos cognitivos, para compor a avaliação final. Analisandose os resultados da validação quanto à interação com o sistema e à adaptação de conteúdo, verificou-se que todos os itens avaliados tiveram resultados positivos com relação ao Ambiente Adaptado. Destaca-se que a $83 \%$ dos alunos que participaram da validação, consideram válida a utilização do sistema implementado. Com relação à adaptação das atividades, $100 \%$ dos alunos consideraram que o tipo de atividade proposta para o seu estilo preferencial favoreceu o processo de aprendizagem. Quanto aos estilos de aprendizagem diagnosticados, $83 \%$ afirmam que a forma de apresentação dos conteúdos favoreceu a aprendizagem.

O estudo realizado por (MÜHLBEIER, 2010) abordou dezessete dimensões de estilos de aprendizagem, implementando um sistema computacional que detecta o estilo preferencial e recomenda diferentes estratégias de aprendizagem para apresentação de materiais educacionais e recursos pedagógicos conforme as preferências individuais dos discentes.

O estilo de aprendizagem do aluno foi identificado através do instrumento "Questionário" embasado nos instrumentos de investigação propostos por Butler (2003), Felder e Silvermann (1993), Honey e Mumford (2000) e Kolb (1984). O resultado do teste identificou as preferências em relação as seguintes dimensões: Acomodador, Analítico, Assimilador, Ativo, Convergente, Divergente, Global, Intuitivo, Pessoal, Pragmático, Realista, Reflexivo, Sensorial, Sequencial, Teórico, Verbal, Visual.

O sistema foi constituído por cinquenta questões objetivas (três questões de cada dimensão). O SDLS (System Detector Learning Styles) foi aplicado a setenta discentes e docentes integrantes das modalidadades presenciais e a distância. Participaram da pesquisa discentes dos Curso de graduação presenciais Ciências Contábeis (16\%), Medicina Veterinária (14\%), Pedagogia (13\%) e o curso à distância de Capacitação Moodle (57\%).

Após a tabulação dos estilos de aprendizagem predominantes foram definidos Métodos e estratégias de ensino (Apresentação de conteúdo, atividades propostas, ferramentas e estratégia pedagógica).

As Pesquisas descritas foram validadas por docentes e discentes dos cursos de Graduação e Pós- Graduação das modalidades presenciais e a distância. Após análise sobre as características, estado e preferência das diferentes dimensões de estilos de aprendizagem 
integrantes dos trabalhos realizados pode-se confirmar a semelhança entre ambas integrantes dos Modelos já citados. A seção a seguir aborda um estudo de caso apresentando as diferentes dimensões de estilos de aprendizagem, suas características e semelhanças embasadas nas pesquisas desenvolvidas.

\section{Resultados e discussão}

Todo o processo educativo é, via de regra, influenciado pelas pessoas envolvidas (professores, alunos), por suas ações e por pressupostos teóricos vigentes na sociedade e na época em que estão inseridos.

Partindo do problema de pesquisa, as questões que serviram para nortear o estudo mostraram-se adequadas no encaminhamento das soluções possíveis para a investigação proposta.

Desenvolveu-se um estudo de caso através de uma análise qualitativa, envolvendo o método de análise de conteúdo embasada nas pesquisas de (MÜHLBEIER, 2010) e (MOZZAQUATRO, 2010).

Desse modo, os registros obtidos dos sujeitos participantes da pesquisa orientou a análise, permitindo assim desenvolver várias conclusões sobre o tema em questão.

$\mathrm{Na}$ pesquisa desenvolvida constatou-se que existe uma tendência a mesclar estilos, confirmando o que diferentes autores (Bariani, 1998) e (Geller, 2004) têm concluído em relação aos estilos de aprendizagem estarem imbricados, conforme mostram as Figuras 1 e 2.

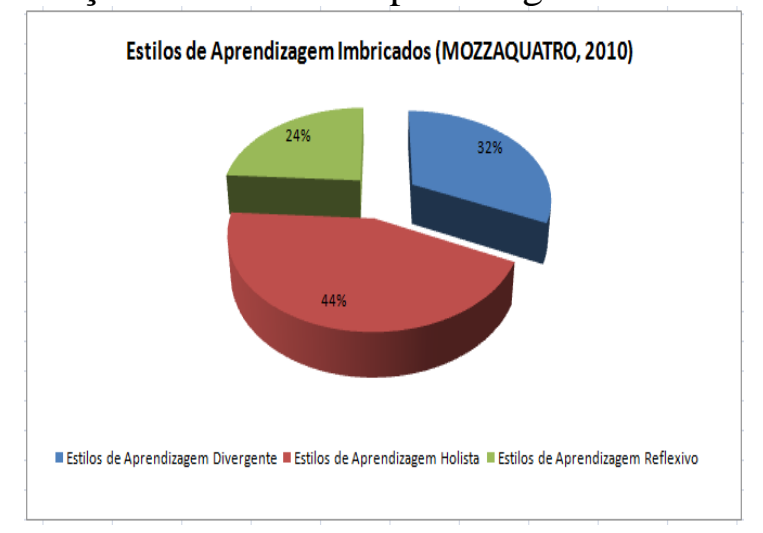

Figura 1 - Estilos de aprendizagem imbricados : Divergente - Holista - Reflexivo.
Estilos de Aprendizagem Imbricados (MÜHLBEIER, 2010)

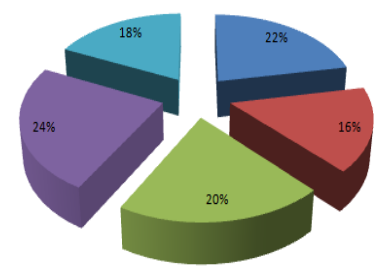

Estios de Aprencizagem Reflexivo IIEstio de Aprendizagem Verba EEstilo de Aprendizagem Visual

Figura 2 - Estilos de aprendizagem imbricados: Reflexivo- Seqüencial - Sens orial - Verbal -Visual

Conforme observa-se na Figura 1, têm-se três estilos imbricados, ou seja, os resultados da população entrevistada na pesquisa de Mozzaquatro (2010) mostram que os indivíduos apresentam predominância nos seguintes estilos: Estilo Holista (44\%), Estilo Divergente (32\%) e Estilo Reflexivo (24\%).

A Figura 2 ilustra a análise da pesquisa desenvolvida por (MÜHLBEIER, 2010) que apresenta a possibilidade do aluno apresentar estilo de aprendizagem predominante imbricado, ou seja, ter mais de um estilo predominante em igual ordem de importância. Podese observar que a população entrevistada apresentou cinco estilos de aprendizagem imbricados: Verbal (24\%), Reflexivo (22\%), Seqüiencial (20\%), Visual (18\%) e Sensorial $(16 \%)$. 
Conforme a pesquisa realizada tratando-se de estilos imbricados pode-se constatar que toda pessoa possui mais de um estilo de aprendizagem, possuindo apenas uma com maior predominância.

Tratando-se das diferentes dimensões dos estilos de aprendizagem integrantes dos Modelos de (WITKIN et al., 1981), (Riding; Rayner, 1998), (Bariani, 1998), (Felder Silverman, 1993), (Honey;Munford, 2000), (Butler, 2003), (Kolb,1984) constatou-se que existem semelhanças entre as características e comportamentos integrante das mesmas.

Conforme observa-se na Figura 3, o estilo de aprendizagem Global abordado por Felder e Silvermann apresenta como enfoque a visão do todo. Comparando o estilo citado ao Estilo de aprendizagem Holista integrante do Modelo de Bariani constatou-se que ambos apresentam características semelhantes tais como ênfase ao contexto global. Partindo-se deste pressuposto conclui-se que um indivíduo com estilo de aprendizagem Holista tem as mesmas preferências relacionadas ao Estilo Global.

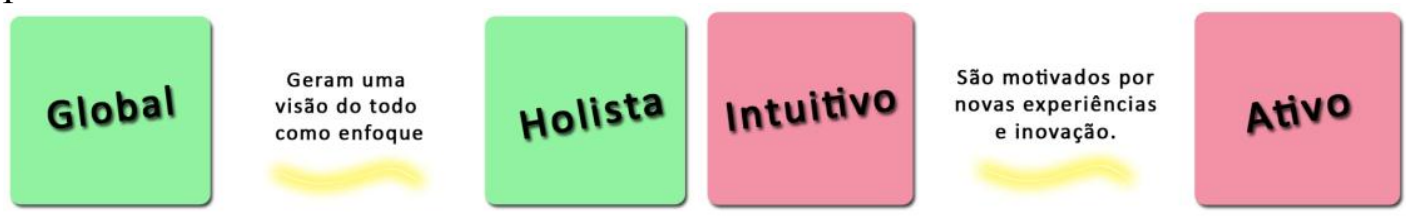

Figura 3 - Semelhança Global e Holista

Figura 4 - Semelhança Intuitivo e Ativo

A Figura 4 ilustra os estilos de aprendizagem intuitivo e ativo integrantes do Modelo Felder e Silvermann, os quais mencionam caracteristica individuais como motivação por novas experiências e inovação. Conclui-se assim que pessoas com tais caracteristica podem se adequar a ambas dimensões.

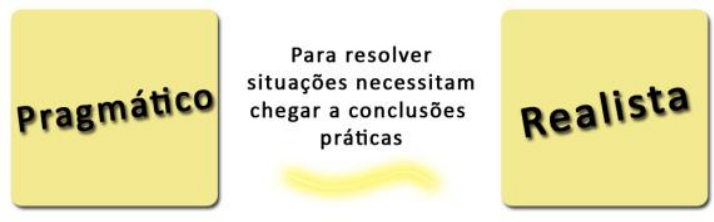

Figura 5 - Semelhança Pragmático e Realista

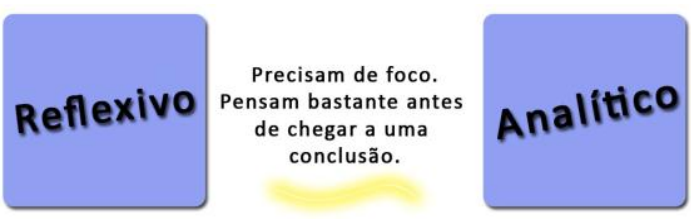

Figura 6 - Semelhança Reflexivo e Analítico

O estilo realista integrante do Modelo de Buther apresenta como caracteristica resolver situações práticas. O Modelo de Honey e Mumford aborda a dimensão pragmático que integra experimentos e teorias aplicadas a prática. Neste contexto contatou-se que ambas as dimensões apresentam semelhança entre si, conforme Figura 5. A Figura 6 ilusta as dimensões Analítico (Modelo de Buther) e Reflexivo (Modelo de Honey e Mumford), ambas apresentam como caracteristica "foco", isto é, individuos reflexivos ou analiticos pensam bastante antes de chegar a uma conclusão.

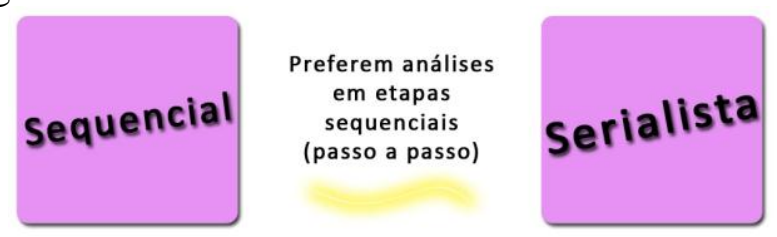

Figura 7 - Semelhança entre as dimensões Sequencial e Serialista.

Conforme a Figura 7 são apresentados respectivamente os estilos de aprendizagem sequencial e serialista integrantes dos modelos de Felder - Silvermann e Bariani apresentando 
como preferências individuais análises em etapas sequenciais (passo a passo). Pode-se constatar que individuos sequenciais são serealistas.

Após análise relacionando as semelhanças entre as dimensões citadas pode-se comprovar que para adaptar um ambiente virtual de aprendizagem, construir um sistema que detecte estilos de aprendizagem ou um laboratório virtual não é necessário utilizar uma grande gama de dimensões, visto que ambas apresentam semelhanças, influenciando positivamente na relação custo beneficio.

\section{Considerações Finais}

Acredita-se que a pesquisa apresentada tenha alcançado seus objetivos, bem como contribuído para uma evolução nas pesquisas sobre o tema adaptação de AVAs aos diferentes estilos de aprendizagem. A principal contribuição deste trabalho foi comprovar a semelhança entre as diferentes dimensões dos estilos de aprendizagem abordadas no modelos de: (WITKIN et al., 1981), (Riding; Rayner, 1998), (Bariani, 1998), (Felder - Silverman, 1993), (Honey;Munford, 2000), (Butler, 2003), (Kolb,1984) constatando-se que não se justifica a utilização de uma variada gama de dimensões de estilos de aprendizagem na adaptação de AVAs.

Com a pesquisa desenvolvida pôde-se constatar a existência de estilos imbricados, ou seja, toda pessoa apresenta predominância em uma dimensão, mas integra outras em menor proporção.O estudo realizado evidencia que diagnosticar estilos de aprendizagem é uma tarefa complexa, e requer análises aprofundadas para que possa ser oferecida ao aluno uma experiência de aprendizagem individualizada, apresentando-the a informação de maneira personalizada e direcionada. A identificação dos estilos de aprendizagem predominantes passa a ter um papel fundamental para viabilizar práticas educacionais mais condizentes com ações que priorizem a autonomia e a cooperação em um processo de ensino e aprendizagem.

Por fim, este trabalho procurou comprovar que ao trabalhar com estilos de aprendizagem deve-se realizar uma análise aprofundada sobre as características integrantes de cada dimensão para assim, identificar semelhanças entre ambas, abordando apenas os estilos de aprendizagem diferenciados. Foi com grande interesse e curiosidade que constatamos, ao longo de todo o processo de desenvolvimento dessa pesquisa, a existência de espaço para desencadear outras pesquisas nessa área. Além desses itens já concluídos, outros aspectos surgiram durante o processo tornando-se inquietações que originarão novas investigações por parte dos pesquisadores, tais como desenvolvimento de um instrumento de mensuração de estilos de aprendizagem unificado integrando os modelos já citados, ou seja, abordando apenas dimensões diferenciadas.

\section{Referências}

BARIANI, I. C. Estilos Cognitivo de Universitários e Iniciação Cientifica. Campinas: UNICAMP. Tese (Doutorado em Educação), Faculdade de Educação, Universidade Estadual de Campinas, 1998.

BUTHER, K. A.. Estilos de Aprendizagem: as dimensões psicológica, afetiva e cognitiva. Traduzido por Renata Costa de Sá Bonotto e Jorge Alberto Reichert. Porto Alegre: Editora da UFRGS, 2003. 
CAVELluCCI, L. C. B.. Estilos de Aprendizagem: em busca das diferenças individuais. 2006, p.10-12. Disponível em:<http://www.iar.unicamp.br/disciplinas/am 540_2003/lia/estilos_de_aprendizagem.pdf>. Acesso em: out. 2011.

DUNN, R. et al Survey of Research on Learning Styles. Educational Leadership, USA, v.46, n.6, p. 50-58, Dec. 1989.

GELLER, M. "Educação a Distância e Estilos Cognitivos: construindo um novo olhar sobre os ambientes virtuais". Porto Alegre: UFRGS. Tese (Doutorado em Informática na Educação), Programa de Pós-Graduação em Informática na Educação, Universidade Federal do Rio Grande do Sul, 2004.

HODGINS, H. W. The future of learning objects, 2000. Disponível em <http//www.reusability.org/read/chepters/hodgins.doc>. Acesso em: set. 2011.

HONEY, P.; I Mumford. A. The Learning Styles helper's guide. Maldenhead Berks: Peter Honey Publications, 2000.

KOLB, D. A.. Experiential Learning: experience as the source of learning and development. Englewood Cliffs: Prentice-Hall, 1984.

MOZZAQUATRO, P. M. Adaptação do Mobile Learning Engine Moodle (MLE Moodle) aos Diferentes Estilos Cognitivos Utilizando Hipermídia Adaptativa. Dissertação de Mestrado - Universidade Federal de Santa Maria (UFSM), 2010.

MÜHLBEIER, A. R. K. Estilos e Estratégias de Aprendizagem Personalizadas a Alunos das Modalidades Presenciais e a Distância. Trabalho de Conclusão de Curso Universidade de Cruz Alta (UNICRUZ), 2010.

SOLOMAN, B. A. ; FELDER, R. M. Index of Learning Styles Questionnaire. Department of Chemical Engineering North Carolina State University Raleigh, NC 276957905,1993. Disponível em: <http $/ /$ www.engr.ncsu.edu/learningstyles/ilsweb .html $>$. Acesso em: out. 2011.

PALLOFF, R.; PRATT,K. The virtual student: A profile and guide to working with online learners. San Francisco: Jossey- Bass Publishers, 2003.

PENNINGS, A. H.; SPAN, P. Estilos Cognitivos e Estilos de Aprendizagem. In: Almeida, L. (org.). Cognição e Aprendizagem Escolar. Porto: APPORT, 1991, p. 120.

RIDING, R. e RAYNER, S. Cognitive Styles and Learning Strategies Understanding style differences in learning and behavior. David Fulton Publisher London, UK, 1998.

WITKIN, H. A.; GOODENOUGH, D. R. Cognitive Style: essence and origins. New York: International Universities Press., 1981. 